\title{
Modelling the Creative Process and Cycles of Feedback
}

\author{
Carol R. Aldous \\ College of Education, Psychology and Social Work, Flinders University, Adelaide, Australia \\ Email: carol.aldous@flinders.edu.au
}

How to cite this paper: Aldous, C. R. (2017). Modelling the Creative Process and Cycles of Feedback. Creative Education, 8, 1860-1877.

https://doi.org/10.4236/ce.2017.812127

Received: August 4, 2017

Accepted: September 23, 2017

Published: September 26, 2017

Copyright (C) 2017 by author and Scientific Research Publishing Inc. This work is licensed under the Creative Commons Attribution International License (CC BY 4.0).

http://creativecommons.org/licenses/by/4.0/

\begin{abstract}
Dual physicist and psychologist Shaw (1989) published an account speculating on the modelling of a series of feedback loops within the structure of the creative process following a series of in-depth interviews with 12 scientists concerning the use of cognitive and affective processes in the act of scientific creation. These feedback loops were hypothesized to operate between each phase of the classical model of creative problem solving as typified by the seminal work of Wallas (1926). However, data providing evidence of their operation has been scarce. Using a sizable sample $(\mathrm{n}=405)$ and recently developed software that enables bidirectional pathways to be modelled (M-Plus v6), this study examines the evidence for the existence of feedback loops within the creative process using structural equation modelling (SEM) procedures. The research builds on the findings of a previous study in which participants solved two novel mathematics problems in the Mathematics Challenge for Young Australians. Empirical evidence for both the classical stage model of creative problem solving and Shaw's feedback loops namely the Vinacke and Lalas loops, are presented and discussed. The participants were middle school students in Grades 7 to 10 drawn from across two states of Australia.
\end{abstract}

\section{Keywords}

Creative Process, Feedback Loops, Bidirectional Modelling, Cognitive, Non-Cognitive

\section{Introduction}

Some 25 years ago, Shaw (1989) described a structure for modelling a creative situation that focused on thoughts as well as feelings. In so doing, Shaw was seeking to model a set of unified processes both cognitive and non-cognitive associated with the modelling of creative behaviour that could be generalized 
across individuals. The inclusion of both cognitive and emotional features within a structural model was forward looking at the time, particularly since cognitive neuroscience today is revealing just how much thinking and feeling are inextricably linked (Damasio, 1994; 1999; Immordino-Yang \& Faeth, 2010).

Numerous models of the creative process have been propounded over time (Lubart, 2001) with many building on the seminal, classical four stage model of preparation, incubation, illumination and verification put forward by Wallas (1926) early in the last century. Some models have sought to elaborate upon the number of stages involved in creativity as for example: (a) Shaw's (1989) model of the "Eureka process"; (b) Osborn's (1953) model of the "Applied imagination"; (c) the "Osborn-Parnes" model of creative problem solving (Isaksen \& Parnes, 1985); (d) the "Core processes" model by Mumford and associates (1991); (e) the "Componential" model by Amabile (1996); and (f) the "Extended phase" model by Cropley and Cropley (2008) to state but some of these. Each of these models has expounded on at least five stages with two sets of scholars advocating as many as eight (viz., Cropley \& Cropley, 2008; Mumford, Mobley, \& Uhlman, 1991). Conversely, other process models have reduced the number of stages, collapsing them instead into a broader set of categories such as that proposed by the "Two tier" model advocated by Runco and Chand (1995) and the "Geneplore" model advanced by Finke, Ward and Smith (1992). Emphases in these models were given to a whole collection of sub-processes hypothesized to be involved in the act of creation. Interestingly, of the process models cited, only two of them dealt explicitly with "feeling" and "affect". These were Cropley's (2001) earlier "Holistic" model of creativity and Shaw's model of the "Eureka process".

As with others before him (e.g. Ghiselin, 1963; Hadamard, 1945; Wallas, 1926) Shaw argued intuitively that the development of a unified model which provided additional information about both cognitive and affective components was strategically important to future understanding and teaching about creativity and the processes of scientific discovery. Having an abiding interest in how scientists created, Shaw set about by means of an interview strategy to identify and describe the experiences and processes that were common to scientists and engineers alike during an act of scientific creation in order to model mathematically some phenomenon in a real world situation.

Of note was the speculation by Shaw of five feedback loops associated with the classical stage model of creative problem solving. These loops involved a series of couplings or feedback cycles arising between each stage of the creative process. The cycling: (a) that arose between the stages of preparation and incubation was called the "Areti Loop" Arieti (1976); (b) that occurred between the stages of incubation and illumination was termed the "Vinacke loop" Vinacke (1952); (c) that arose between the stages of illumination and elaboration (or explication) was called the "Lalas loop"; and (d) the coupling between elaboration and the final outcome (or creative synthesis) was termed the "Communication 
loop". Finally a series of models of feedback loops arising between the final outcome (or the creative synthesis) and each earlier stage in the model of creative problem solving was named the "Rossman loop" (Rossman, 1931). Where practicable Shaw's naming of each feedback loop was based according to the name of the researcher thought to be responsible for theorizing its presence (Shaw, 1989).

In addition Shaw modelled a set of affective states both positive and negative depending on stage and circumstance associated with each loop. Further, it was proposed that the Areti and Vinacke loops modelled movement between conscious and non-conscious processing, since the stage of incubation was a situation marked by non-conscious activity in which the problem at hand was set aside for a time. Although Shaw hypothesized the presence of five feedback loops, he speculated on the presence of many more models. A diagram showing the five feedback loops superimposed onto the classical four stage model of creative problem solving is given in Figure 1.

However to date little or no large scale data has been presented examining the models of these feedback loops. Interestingly, as a physicist, Shaw believed that the processes depicted in the creative structure could be modelled mathematically and foreshadowed the introduction of powerful new computational techniques that would make this possible, citing Chaos Theory (Gleick, 1987) and Catastrophe Theory (Dold \& Eckmann, 1977) as examples. It is regarding the possibility of this latter point that the content of this current paper raises. This modelling is made feasible through the use of recently developed computational software that permits the examination and estimation of the significance of reciprocal paths operating between two components, namely M-Plus Versions 6, 7 and 8 (Muthén \& Muthén, 2010 ; 2012; 2017) to take place. This is essential if the statistical validation and verification of the feedback loops is undertaken. However, even with the appropriate software it is still necessary for the researcher to find suitable raw data in which the hypothesized models can be both advanced and examined.

\section{A Previous Study: Preliminary Examination of Feedback Loops}

In a study of novel mathematics problem solving involving a large population of 405 middle school students participating in the Mathematics Challenge for Young Australians (Aldous 2009; 2015), the preliminary examination of Shaw's feedback loops was found in the outer measurement model of the causal or structural model of creative problem solving constructed using structural equa-

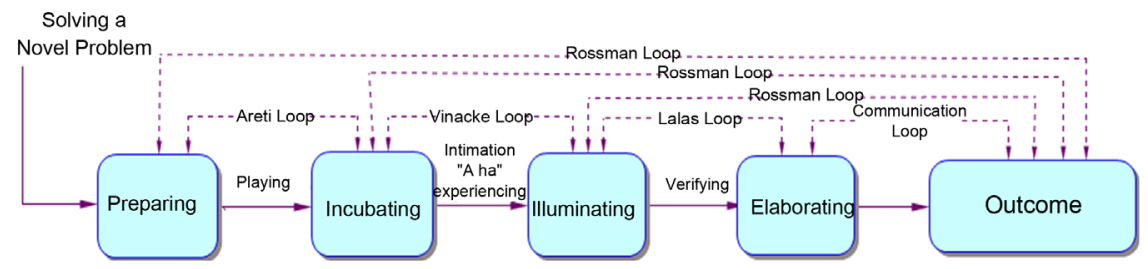

Figure 1. The Classical Model overlaid with Shaw's feedback loops (Aldous, 2012: 845). 
tion modelling procedures (Aldous, 2006; 2014). The purpose of the study was to form a comprehensive model of creative problem solving in which both cognitive and non-cognitive processes used in the creative "process" were modelled along-side other more macroscopic components related to the creative "person", the creative "product" and the creative "environment" relevant to a confluence approach (Sternberg, 2006).

In this situation the participants in the Mathematics Challenge for Young Australians had three weeks in which to solve six novel problems, (the event being a precursor for selection into the Australian Mathematics Olympiad). Consequently, the participants had extended time to incubate on the novel problems and to put into action the heuristic processes first proposed by Wallas (1926) and elaborated on by Shaw (1989). To this end a self-report instrument referred to as the Systems of Reasoning Questionnaire (SRQ) (Aldous, 2001; 2006; Jeffries, 2011) is employed to tap both cognitive and non-cognitive processes used in solving the novel mathematics problems. In its development the SRQ was grounded in Sloman's (1996) two systems of reasoning and Epstein's (1994, 1998) two patterns of mind and was consistent with Kahneman's (2011) System 1 and System 2 thinking. It is used in this study in conjunction with two problems about which students were invited to report concerning use of both their thinking and feeling (intuitive) approaches to reasoning. One problem called the Cute Numbers problem is spatially oriented, the other called the Birthday Cake problem, is more numerically oriented.

The SRQ, comprises a set of five scales referred to collectively as the Approaches to Reasoning. These are a Strategic approach, a Free-flowing approach, a Spatial-verbal approach, a Feeling approach and a Systematic approach to reasoning. The Strategic and the Systematic approaches to reasoning tap cognitive processing while the Free-flowing and Feeling approaches to reasoning tap non-cognitive processing. Interestingly the Spatial-verbal approach to reasoning engages both cognitive and non-cognitive processing depending on whether mainly successive or simultaneous synthesis is being used.

\section{Larger Model Holds Information with which to Test Classical Model}

The comprehensive model of creative problem solving comprises both macroscopic and microscopic components relevant to creativity. The macroscopic component models aspects of the creative person, the creative product and the creative environment. The microscopic component seeks to model the creative processes both cognitive and non-cognitive used in creative problem solving and comprises the Approaches to Reasoning construct. In this way the microscopic process component as measured by the Approaches to Reasoning construct is nested within the larger more macroscopic one, reflecting a more holistic approach to the study of creativity. In effect there is a model within a model.

It is an examination of the highest loading items in the Approaches to Reasoning construct of the outer model of the comprehensive model of creative 
problem solving that evidence of Shaw's feedback loops is indicated. These early data provide evidence of (a) the constructs of preparation cycling into incubation in the Strategic approach to reasoning, (b) the constructs of incubation cycling into insight in the Free flowing approach to reasoning, (c) the constructs of intimation cycling into sudden illumination in the case of the Cute Numbers problem, and intimation cycling into gradual illumination in the Birthday Cake problem in the Feeling approach to reasoning. Finally in the Cute Numbers example the elaboration of findings is indicated by the Systematic approach to reasoning, while the verification of findings is indicated by the Birthday Cake case. Interestingly in the Spatial Verbal approach to reasoning, evidence of the nature of processing involved is found. Predominantly simultaneous processing is indicated in the case of the Cute Numbers problem while sequential processing is indicated for the most part in the case of the Birthday Cake problem. A summary of these early findings is given in Table 1 .

Nevertheless it remains to be seen whether these cycles can be modelled separately in a classical model of creative problem solving (CPS) independent of the larger causal model in which they are initially instituted. Movement in the forward direction provides clear evidence but can movement in the backwards direction also be modelled? In essence can reciprocal paths be located and modelled and if so, what is the meaning of any such cycling?

Using information contained within the larger comprehensive model of creative problem solving (Aldous, 2006; 2014) the following questions are advanced. These are:

Table 1. Preliminary evidence of Shaw's loops.

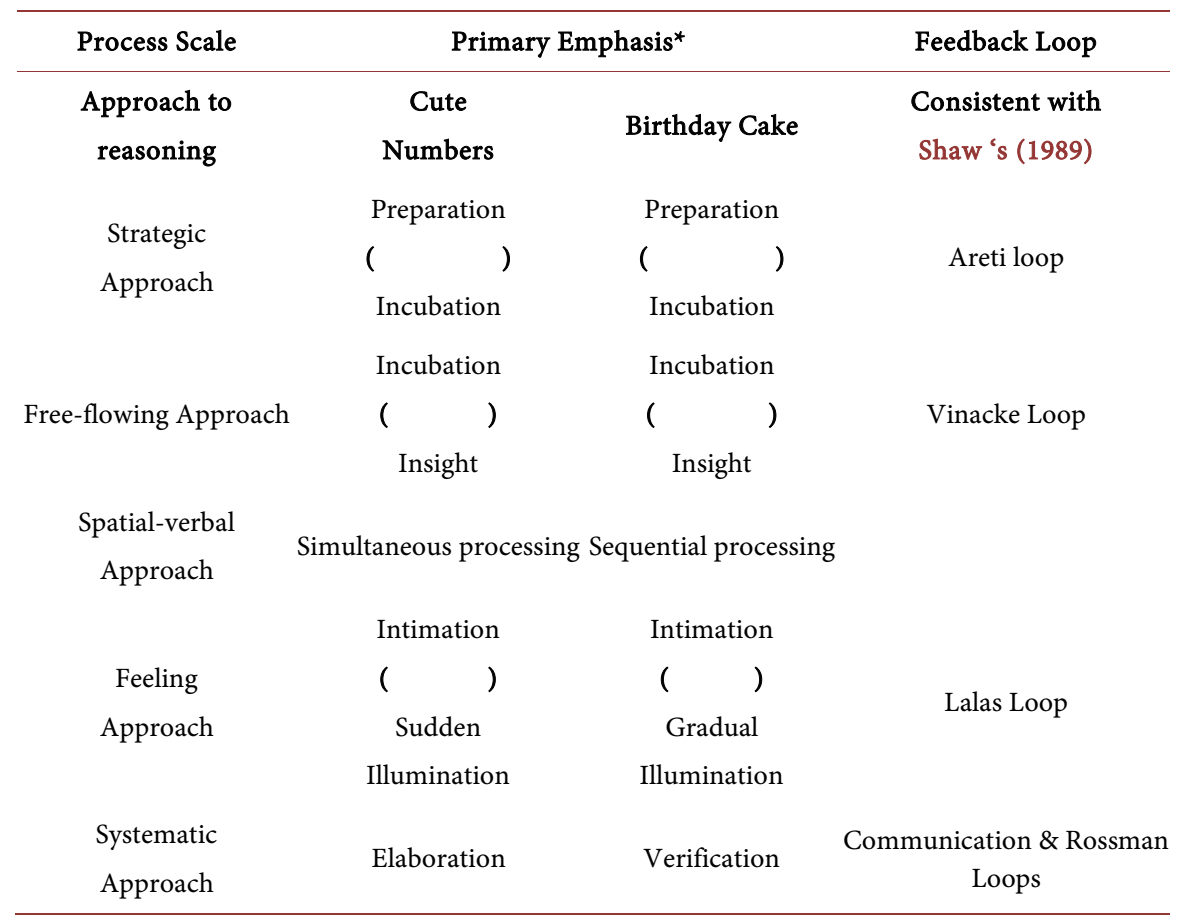

* based on four highest items (refer to Table 2 for specific details). 
1) Can the stages of the classical model of creative problem solving be modelled?

2) Can Shaw's feedback loops be located and modelled empirically?

3) Can the classical model and cycles of feedback be interpreted meaningfully in more than one data set?

\section{Method}

\subsection{Participants}

These comprised a non-random sample of 405 middle school students in Grades 7 to 10 who had enrolled in the Mathematics Challenge for Young Australians (2001) from within 11 independent schools, across two states of Australia. Some of the schools enrolled their entire cohort in the Mathematics Challenge while others allowed students to self-select into the activity. Students from both contexts who agreed to participate in the investigation were employed in this study. The students ranged in age from 11 to 16 years (mean age $=13.9$ years, $\mathrm{SD}=$ 1.0). Of these 199 were female and 206 were male.

\subsection{Instruments}

\subsubsection{Mathematics Challenge for Young Australians}

The Mathematics Challenge for Young Australians is a national event organized by the Australian Mathematics Olympiad in conjunction with the Australian Mathematics Trust to encourage as many young people as possible to engage with enriching mathematics problems. The problems are purposefully designed by a committee to be both novel and challenging (Australian Mathematics Trust, 2001). Students have three weeks in which to solve six novel problems. Two of the six problems were used in this study. They were chosen to coincide with those problems that were common across the grade levels from primary, junior and intermediate stages of schooling in the Mathematics Challenge event.

One problem referred to as the Cute Numbers problem involved a search for pattern in number and the ability to generalize well. It recruited facility in approximate arithmetic, an ability known to utilize the visual spatial circuits of the brain (Dehaene, Spelke, Pinel, Stanescu, \& Tsivkin, 1999). The second problem referred to as the Birthday Cake problem required the use of fractional arithmetic and the systematic application of the formula for the area of a triangle and was largely numerical in nature. However some visualization concerning where to cut the birthday cake was required in order to meet the limitations of the problem.

Not every student provided an answer to both problems. Some students answered the Birthday Cake problem only, while others answered the Cute Numbers problem only, while still others answered both problems. Of the 405 participants involved, 360 answered the Birthday Cake problem and 387 answered the Cute Numbers problem. Scores on each of the problems assigned by the judges of the event were used to form the latent variable Outcome in the classical mod- 
el of creative problem solving under review.

\subsubsection{Systems of Reasoning Questionnaire}

The Systems of Reasoning Questionnaire (SRQ), is designed to tap into cognitive and non-cognitive processes used in creative problem solving within the field of novel mathematics problem solving. It was initially developed by Aldous (2001; 2006 ; 2009) and subsequently validated in a cross case analysis by Jeffries (2011) and Jeffries \& Aldous (2016). It comprises five scales. The Strategic approach comprises ten items related to strategy thinking, the Free flowing approach comprises six items related to semi-conscious and non-conscious behavior during thinking, the Spatial-verbal approach comprises eight items related to simultaneous and sequential spatial and verbal thinking (Das, 2003), the Feeling approach comprises ten items related to promptings by gut feelings, intuition and insight and the Systematic approach comprises ten items concerning logical and systematic thoughts. Participants were asked to complete the SRQ as soon as practicable after solving each novel problem. Respondents answer True (scored two points), False (scored zero points) or Not sure (scored one point) to each item in the questionnaire. Selected items from the SRQ were used to form the latent constructs in the classical model of creative problem solving.

\subsection{Bidirectional Path Modelling with Latent Variables}

\subsubsection{Latent Variables}

Structural equation modelling $S E M$ with latent variables enables variables that are not readily observed to be reflected by one or more indicators which are considered to be manifestations of the latent construct. This is important when investigating multifaceted constructs such as those involved in characterizing the stages of the classical model of creative problem solving. Latent variables formed from multiple variates have been found to have greater reliability and validity than variables singly observed (Sellin \& Keeves, 1997). In this study it is the four highest loading items as identified in the outer model of the comprehensive model of creative problem solving (Aldous, 2006; 2014) contained from among the Approaches to Reasoning constructs that are used in the formation of the latent variables. Specifically, items belonging to (a) the Strategic, (b) the Free-flowing, (c) the Feeling, and the (d) Systematic approaches to reasoning, grounded in the System of Reasoning Questionnaire were used in forming the latent constructs of Preparing, Incubating Illuminating and Elaborating respectively. The statement of items, their cognitive function, the scale to which each item belongs in the comprehensive model, together with the latent variables the items are used to reflect are given in Table 2.

\subsubsection{Bidirectional Modelling}

The examination of bidirectional relationships has rarely been undertaken in the causal analysis of data. However with the development of new software having the capacity to accommodate increased complexity in model specification, esti- 
Table 2. Items used in forming the latent variables of the classical model of CPS.

\begin{tabular}{|c|c|c|c|c|}
\hline $\begin{array}{l}\text { Reasoning } \\
\text { Approach }\end{array}$ & $\begin{array}{c}\text { Latent } \\
\text { Variable }\end{array}$ & Item & Statement of Manifest Variable & $\begin{array}{l}\text { Cognitive } \\
\text { Function }\end{array}$ \\
\hline \multirow{4}{*}{$\begin{array}{l}\text { Strategic } \\
\text { Approach }\end{array}$} & \multirow[t]{4}{*}{ Preparing } & 41 & I tried a lot of different ways until I found the right one & Exploration \\
\hline & & 52 & $\begin{array}{l}\text { I had several attempts at finding a solution before I gave up for a time and } \\
\text { came back to it later }\end{array}$ & Impasse \\
\hline & & 53 & I played around and explored the problem for a while & Exploration \\
\hline & & 56 & I set the problem aside for a time & Impasse \\
\hline \multirow[t]{4}{*}{$\begin{array}{l}\text { Free-flowing } \\
\text { Approach }\end{array}$} & \multirow[t]{4}{*}{ Incubating } & 36 & I drew upon all mental resources/parts of me to solve this problem & Breadth of attention \\
\hline & & 27 & I connected with the patterns in this problem & Associative \\
\hline & & 57 & $\begin{array}{l}\text { Having set aside the problem for a time I found that the solution suddenly } \\
\text { popped into my mind }\end{array}$ & $\begin{array}{l}\text { Associative } \\
\text { illumination }\end{array}$ \\
\hline & & 39 & $\begin{array}{l}\text { I thought of how to get the answer to this problem while I was doing } \\
\text { something else (e.g. riding a bike) }\end{array}$ & $\begin{array}{l}\text { Defocused } \\
\text { attention }\end{array}$ \\
\hline \multirow[t]{4}{*}{$\begin{array}{l}\text { Feeling } \\
\text { Approach }\end{array}$} & \multirow[t]{4}{*}{ Illuminating } & 38 & I developed a feeling about the correctness of my solution before I checked it & $\begin{array}{l}\text { Intra-personal } \\
\text { scrutiny }\end{array}$ \\
\hline & & 28 & I had a sense of relative sizes in this problem & Cue relevance \\
\hline & & 22 & $\begin{array}{l}\text { The 'answer' or 'how to get the answer' suddenly came into my head while I } \\
\text { was working on this problem }\end{array}$ & Intuitive insight \\
\hline & & 55 & I followed a feeling/ hunch about what to do & Intuition \\
\hline \multirow[t]{4}{*}{$\begin{array}{l}\text { Systematic } \\
\text { Approach }\end{array}$} & \multirow[t]{4}{*}{ Elaborating } & 31 & I used a sequence of logical steps in this problem & $\begin{array}{l}\text { Sequential } \\
\text { reasoning }\end{array}$ \\
\hline & & 29 & I organized my reasoning/thinking in a strategic way & Organizing \\
\hline & & $49 \mathrm{~h}$ & Talked over the quantities in your mind & Familiarization \\
\hline & & 34 & I tried to verify/check that my answer was correct & Evaluation \\
\hline
\end{tabular}

mation of reciprocal effects in path models is now available (Razak, Keeves, \& Darmawan, 2014) Consequently, the latent variables constructed in this study are used to investigate the classical model of creative problem solving both in the forward direction and in the backward direction operating simultaneously.

The bidirectional modelling is examined with maximum likelihood estimation procedures using the M-Plus (Version 6.0.) computer program (Muthén \& Muthén, 2010). On each occasion replication of the testing occurs twice, once with the Birthday Cake data and once with the Cute Numbers data.

\subsection{The Modelling Procedure}

Following evidence that the larger comprehensive model of creative problem solving (CPS) holds information with which to test the classical model the following issues are specified for testing the classical model of creative problem solving in each data set.

Issue One: Can the classical constructs of CPS be specified and identified and forward paths modelled? 
Issue Two: Can reciprocal paths be modelled and if so what direction (or sign) do the paths take?

Issue Three: What is the meaning of the bidirectional paths and what does this imply for further modelling?

\section{Results}

\subsection{Issue 1: Forward Paths: Can Classical Constructs of CPS be Modelled?}

The M-Plus path models (showing the complete inner and outer paths) of the classical model of creative problem solving, specified in the forward direction for both the Cute Numbers and the Birthday Cake data are given in Figure 2. Unstandardized or metric estimates are shown since these are recommended for making comparisons across different data sets (Kline, 1998) in the initial phase of model construction. Approximate standard errors are given in brackets. The Root Mean Square Error of Approximation (RMSEA) of 0.064 having a 90 per cent confidence interval with a lower bound of 0.057 and an upper bound of 0.07 indicates good model to data correspondence (Hu \& Bentler, 1999). The Standardized Root Mean Square Residual (SRMR) of 0.078 also represents a favourable indication of model fit (Kline, 1998). Although a non-significant chi-square is not achieved the normed chi-square given by the ratio of chi-square divided by the degrees of freedom (Jöreskög, 1969) of 2.5 is indicative of a well-fitting model (Kline, 1998).

\subsubsection{Outer Model or Measurement Model}

From a visual inspection of Figure 2, the metric loadings that form the outer paths in each model can be considered to be the same for both the Cute Numbers and Birthday Cake data. In both models, all outer model paths are significant. This permits meaningful comparisons of the inner paths between models to be made. Further a mean $\mathrm{R}^{2}$ of 0.32 (effect size $f^{2}=0.47$ ) is obtained for both
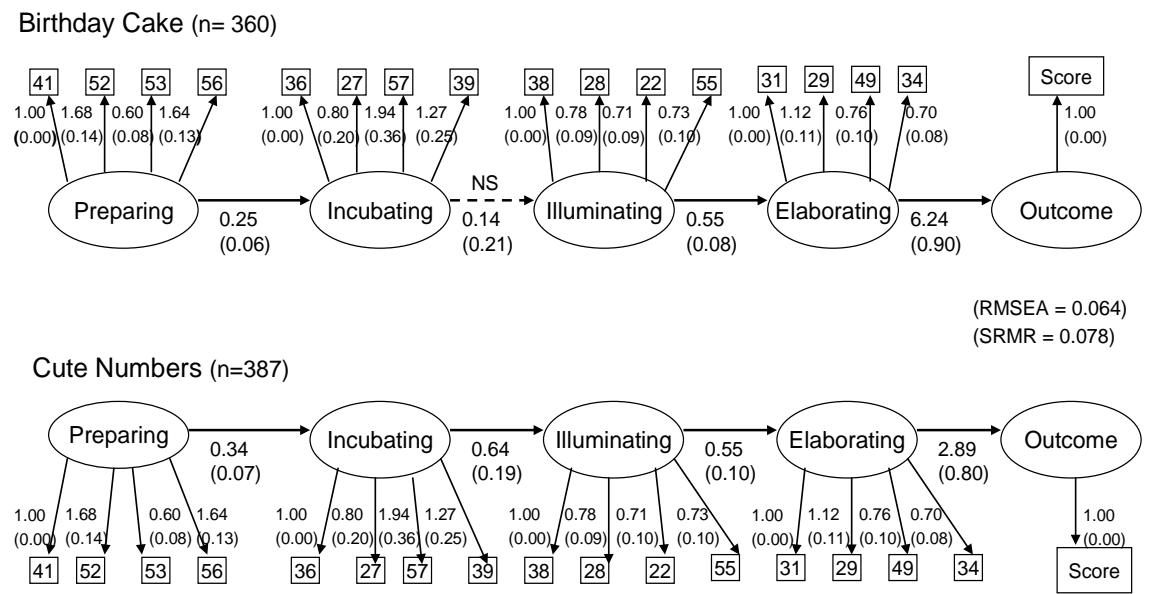

Figure 2. Path diagrams of the Classical Model of creative problem solving. Non-standardized estimates recorded. 
the Cute Numbers and Birthday Cake models indicative of a large effect size (Cohen, 1992: 157) and robust outer models.

\subsubsection{Inner Model or Structural Model}

As can be seen in Figure 2, all inner model paths are positive and significant in both the Cute Numbers and Birthday Cake models with one exception. The path leading from Incubating to Illuminating is not significant in the Birthday Cake model. The critical ratio (CR) defined by the ratio of the unstandardized estimate to standard error is only 0.66 .

Path estimates with a CR larger than 1.96 are significant at the five per cent level, however paths with a CR between 1.64 and 1.96 are significant at the ten per cent level (Kline, 1998). Nevertheless, a mean $\mathrm{R}^{2}$ of 0.24 (effect size $f^{2}=0.31$ ) for the Birthday Cake model indicates an inner model with a medium to large effect (Cohen, 1992: 157) while a mean $\mathrm{R}^{2}$ of 0.20 (effect size $f^{2}=0.25$ ) for the Cute Numbers model indicates an inner model of medium strength effect (Cohen, 1992: 157). Thus adequate stability in the estimation of the models is indicated and a sound explanatory effect for the classical model of CPS is signified.

\subsection{Issue 2: Reciprocal Paths: Can Bi-Directional Paths be Modelled?}

The M-Plus path results for the classical model of creative problem solving, modelled with bidirectional paths for both the Cute Numbers and the Birthday Cake data are given in Figure 3. Standardized estimates are shown since these are recommended for making within model comparisons (Kline, 1998). Standard errors are given in brackets. Significant paths are indicated with solid lines, non-significant paths with dashed lines. Non-significant paths that could not be modelled are shown with a dotted line. As with the Issue One forwardpath models, the RMSEA is 0.064 having a 90 per cent confidence interval with a lower bound of 0.058 and an upper bound of 0.07 , an SRMR of 0.071 and a normed chi-square of 2.5 indicating good model to data correspondence for the classical model of creative problem solving that incorporates bidirectional paths ( $\mathrm{Hu} \&$

Birthday Cake $(n=360)$

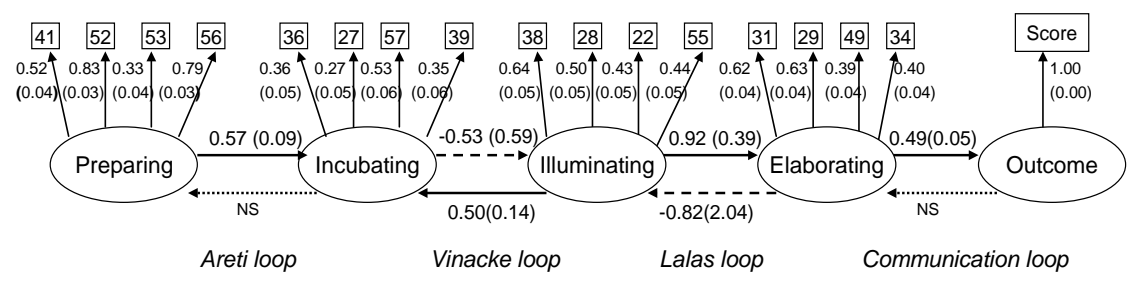

Cute Numbers $(n=387)$

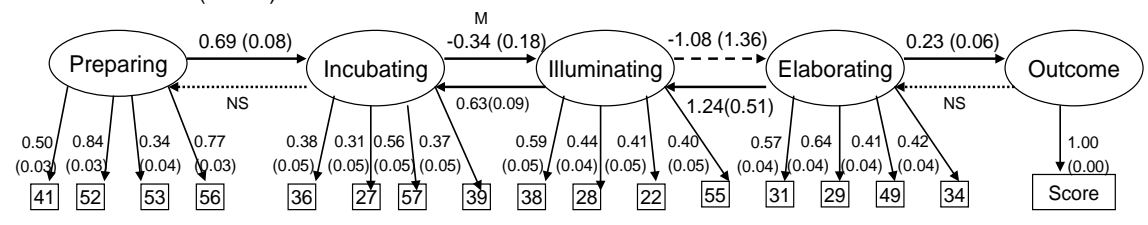

Figure 3. Path diagrams showing bidirectional paths. Standardized estimates recorded. 
Bentler, 1999).

\subsubsection{Outer Model or Measurement Model}

As with Issue One models all outer model paths are significant and the mean $\mathrm{R}^{2}$ of 0.34 (effect size $f^{2}=0.47$ ) again indicates that the outer models are robust (Cohen, 1992: 157). A visual inspection indicates that as with the unstandardized estimates, the standardized estimates in both models are comparable in magnitude.

\subsubsection{Inner Model or Structural Model}

As with the previous modelling the mean $\mathrm{R}^{2}$ for the Birthday Cake inner model is 0.24 (effect size $f^{2}=0.31$ ) signifying a medium to large effect (Cohen, 1992: 157) while the mean $\mathrm{R}^{2}$ for the Cute Numbers inner model is 0.20 (effect size $f^{2}$ $=0.25$ ) indicative of medium strength effect (Cohen, 1992: 157).

Concerning the modelling of feedback between the phases of Illuminating and Elaborating congruent with the Lalas loop, the forward path is positive and significant in the Birthday Cake problem but negative and not significant in the Cute Numbers problem. By way of contrast the backward path is positive and significant in the Cute Numbers problem, but negative and not significant in the Birthday Cake problem.

\subsection{Issue 3: Reciprocal paths: Meaning of the Bi-Directional Paths?}

It can be stated that although the magnitude of the inner paths in both the forward and backward direction for each model falls within the medium to large effect size range (Cohen, 1992), the size of some standard errors indicates that some reverse paths are not significant. Nevertheless the sign or direction of each path remains to be interpreted for each loop.

\subsubsection{Vinacke Loop}

Concerning the modelling of the Vinacke loop, the negative sign in the forward direction arising between the stages of Incubating and Illuminating in both problems indicates that the longer the period of incubating the shorter or more sudden the illuminating phase. Alternatively, it may also be stated that the shorter the incubating period, the longer or more gradual the illuminating phase. The negative sign in the forward direction in both the Cute Numbers and Birthday Cake models signifies that this interpretation is consistent across data sets.

However, concerning the modelling of the Vinacke loop in the backward direction, the positive sign in both the Cute Numbers and Birthday Cake data indicates that in the process of feedback a slower or longer illuminating period cycles with a longer incubating period. Alternatively during feedback a shorter or more rapid illuminating period cycles with a shorter incubating period.

\subsubsection{Lalas Loop}

Concerning the Lalas loop the forward paths in both the Birthday Cake and Cute 
Numbers problems are opposite in sign. A positive and significant path between the stage of Illuminating and Elaborating is indicated in the Birthday Cake problem but a negative and non-significant path is indicated in the forward direction of the Cute Numbers problem. With respect to the forward path in the Birthday Cake problem a longer or more gradual period of illuminating leading to a longer period of elaborating is indicated. By contrast in the Cute Numbers problem a longer and more gradual period of illuminating results in a shorter period of elaborating, alternatively, a shorter period of illuminating results in a longer period of elaborating. However this path is not significant in the Cute Numbers case.

Concerning the interpretation of the backward path in the Lalas loop, contrasting signs in the Birthday Cake and Cute Numbers problems are indicated. While the loading is positive and significant in the Cute Numbers problem, the loading is negative and non-significant in the Birthday Cake example. The positive loading in the feedback cycle of the Cute Numbers problem indicates that a longer period of elaborating cycles to a longer or more gradual period of illuminating, alternatively a shorter period of elaborating cycles to a shorter or more rapid period of illuminating when solving the Cute Numbers problem. However the negative loading in the case of the Birthday Cake problem signifies that a longer period of elaborating may cycle to a shorter period of illuminating, or alternatively a shorter period of elaborating may cycle to a longer period of illuminating. However this backward path is not significant in the Birthday Cake problem.

The sign and significance of the loadings for the forward and backward paths in each of the Vinacke and Lalas Loops for the Birthday Cake and Cute Numbers data are presented in Table 3.

\section{Discussion}

This study commences by asking three questions. The first question concerning whether the stages of the classical model of creative problem solving can be modelled is answered in the affirmative as is evident in the data from the Cute Numbers problem and with all but the exception of one path in the Birthday

Table 3. The sign and significance of loadings in the Vinacke and Lalas Loops.

\begin{tabular}{ccccc}
\hline & Vinacke & Loop & Lalas & Loop \\
\hline Cute Numbers & Birthday Cake & Cute Numbers & Birthday Cake \\
Forward path & & & & \\
significant & Yes & No & No & Yes \\
sign & $(-)$ & $(-)$ & $(-)$ & $(+)$ \\
Backwards path & & & & \\
significant & Yes & Yes & Yes & No \\
sign & $(+)$ & $(+)$ & $(+)$ & $(-)$ \\
\hline
\end{tabular}


Cake problem. This exception is likely to reflect that the relative importance of different cognitive and non-cognitive processing is required in order to solve successfully the problem.

The second question, regarding whether Shaw's feedback loops can be located and modelled empirically may be answered by stating that while evidence for the Areti and Communication loops cannot be located empirically in the current data, evidence for the feedback processes indicative of the Vinacke and Lalas Loops are found in both the Cute Numbers and Birthday Cake data. Nevertheless these findings cannot be used to infer that the Areti and Communication feedback loops are not in operation in solving the two problems, only that the data used in this study are unable to estimate the relationships. Indeed the cycling pattern of exploration and impasse within the items forming the latent variable Preparing indicates feedback consistent with the Areti loop being present. Interestingly studies conducted by creativity researcher Patrick (1938) early last century, showed overlap between stages and that stages such as preparation and incubation might co-occur (as cited in Lubart, 2001). Consequently empirical confirmation of the Areti loop as well as the Communication loop, awaits further trialling. Furthermore, due to difficulties with convergence, attempts to model the bidirectional paths relating to the Rossman loops are not undertaken at this time.

The third question concerning whether the creative heuristic and cycles of feedback can be meaningfully interpreted in more than one data set may also be answered in the affirmative. The discussion that follows in explanation of this statement considers the interpretation of the bidirectional paths, first in the Birthday Cake data and then subsequently in the Cute Numbers data. Interestingly, it was with data obtained from a larger comprehensive model of creative problem solving (Aldous, 2009; 2014; 2015) that modelled both macroscopic and microscopic components of creativity, that turned out to be the most useful in making sense of Shaw's feedback loops. This begs the question what is the articulation between the macroscopic elements of creativity related to the person, environment and product and its more microscopic elements related to process that permitted recursion and cycles of feedback to occur in the creative process?

\subsection{Birthday Cake Data}

The comprehensive model of creative problem solving indicates that the Birthday Cake problem is characterized with a demand for high verbal processing, gradual illumination and successive synthesis. Further, both cognitive and non-cognitive processes are needed to reach a successful solution. Systematic cognitions have a direct effect while feeling cognitions have an indirect effect on the problem solution. Consequently, the finding that the negative forward path of the Vinacke loop, signifying that increased incubation leads to a rapid or short illumination is not significant in the Birthday Cake data and is fully compliant with the finding of the comprehensive model that successful solution in- 
volves a gradual rather than rapid illumination over time in the Birthday Cake problem. However the positive backward path, signifying that a long and gradual period of Illuminating is indicative of a long period of Incubating in the Vinacke loop, is also consistent with the finding of the earlier study that reaching a successful solution in the Birthday Cake problem requires long periods of conscious, systematic and successive synthesis interspersed with extensive periods of non-conscious activity. This result concurs with the interpretation of the positive forward path in the Lalas loop that a slow and gradual illumination leads to a long elaboration which in turn leads to a positive outcome. That the backward path from Elaborating to Illuminating is not significant is also consistent with a meaningful interpretation of the feedback loop, since a negative loading signifies that a long elaboration results in a rapid or short illumination which is found not to be the case in the Birthday Cake problem.

\subsection{Cute Numbers Data}

By way of contrast to the Birthday Cake problem, data obtained from the comprehensive model of creative problem solving indicate that the Cute Numbers problem is characterized by a high visual-spatial processing demand, sudden illumination and simultaneous synthesis. Further, feeling cognitions have a direct effect and systematic cognitions have little or no effect on successfully solving the problem. Consequently, whereas the negative forward path of the Vinacke loop is not significant in the Birthday Cake problem the negative forward path of the Vinacke loop is significant, albeit at the ten per cent level in the Cute Numbers case since successful solution results from longer incubation leading to a sudden or short illumination. Similarly with the positive backward path in the feedback loop a slow and long illumination results in a return to a second period of incubation before a resolution can subsequently occur by a sudden illumination, cycling once more in the forward direction. Given that in the Cute Numbers problem a solution can be found straight from a feeling of cognition, (skilled intuition) that involves simultaneous synthesis without recourse to a systematic approach, the finding that the forward path from Illuminating to Elaborating is not significant is also consistent with what is known previously about this problem from the comprehensive model. A rapid or sudden illumination does not require a long elaboration indicative of a systematic approach in the Cute Numbers problem, nor is a long gradual illumination leading to a short elaboration signified. However, for the problem solver to find a solution, a long and deliberate elaboration may result in a return to a slow illumination as signified by the backward path of the Lalas loop until such time as a sudden illumination arises and is signified by a brief elaboration. Further while the path from Elaborating to Outcome is positive in the Cute Numbers example its magnitude is approximately half that demonstrated by the Birthday Cake example, once again indicating that a solution arises directly from a sudden illumination without recourse to a long period of verification. 
In comparing the different cognitive and non-cognitive processes at work within the two problems and realizing that the Birthday Cake problem is characterized by gradual illumination and the Cute Numbers problem by sudden illumination it is perhaps interesting to note the comparative study undertaken by Miller (1992) who reported that Einstein's breakthroughs were typified by long gradual illuminations but that the French mathematician Poincarés breakthroughs were typified by sudden illuminations rather than gradual ones.

In a recent account concerning the solution of a proof for Fermat's last theorem that had confounded the world for more than 358 years, evidence of both sudden and gradual illuminations in succession were indicated (Singh, 2011). The successful proof developed by Field medallist Andrew Wiles was a gradual process spanning years and was built on a series of sudden and successive illuminations about where to go next in a long and protracted solving of the problem. Further, periods of concentrated effort were interspersed with periods of relaxation through redirected attention being given to play with his young family (Singh, 2011). Such activities provided opportunity for periods of incubation and non-conscious processing to occur, that was important for successful novel problem solving and the expression of creativity.

Evidence presented in this study indicates a strong involvement of cycling through the Vinacke loop especially for problems requiring a sudden illumination, whereas the importance of feedback in the Lalas loop is implicated in problems requiring more gradual illumination. The role of incubation and non-conscious activity concerning both sudden and gradual illuminations, however, cannot be overstated and has implications for the learning and teaching of scientific creativity. Indeed in a recent study, evidence was presented indicating that the benefits of incubation were due to the work of non-conscious processing rather than other explanations such as a break from functional fixedness, although this may also occur (Gallate, Wong, Ellwood, Roring, \& Snyder, 2012).

Lastly a discussion of findings is not complete without making mention of the study's limitations. Mention is made in this study of the Birthday Cake problem requiring verbal processing while the Cute Number problem requires spatial processing although not exclusively so. No attempt to address this aspect of the problem is made in this study or to investigate what role visual-spatial and or verbal processing may have on recursion and the functioning of the feedback loops. Furthermore, while significant paths are evidenced in both the forward and backwards direction in the Vinacke loop within a single problem namely the Cute Numbers problem, significant paths in both the forward and backwards direction in the Lalas loop are obtained only through a collective combination of evidence from both the Birthday Cake and Cute Numbers problems. The forward path is significant in the Birthday Cake problem and the backwards path is significant in the Cute Numbers problem. Consequently, empirical quantitative evidence for the existence of the Lalas loop while substantial, is inferred rather 
than completely verified.

\section{Conclusion}

This study finds that the modelling of the Vinacke and Lalas feedback loops in the creative process using bi-directional structural equation modelling procedures is both possible and substantial. However there is further work to be done. Of note is the finding that interpreting the specific meaning of the feedback loops requires understanding about the context of the problem and knowledge about the kind of processes both cognitive and non-cognitive that are used to solve the problem. A number of questions arise. How does the creative process vary according to the nature of the problem? What processes, both cognitive and non-cognitive, provoke cycling through the feedback loops and in what sequence does this cycling occur? What processes, both cognitive and non-cognitive, define the latent constructs of preparing, incubating, illuminating and elaborating? In finding and modelling possible solutions to these and other related problems, it may be helpful to remember Guilford's own words that there is "something creative about all genuine problem solving" (Guilford, 1967: 312).

\section{References}

Aldous, C. R. (2001). Measuring Cognitive and Non-Cognitive Systems of Reasoning: Some Preliminary Findings. International Education Journal, 2, 1-18.

Aldous, C. R. (2006). Creativity in Problem Solving: Uncovering Cognitive and Non-Cognitive Systems of Reasoning in the Solving of Novel Mathematics Problems. Ph.D. Thesis, Adelaide: The Flinders University of South Australia.

Aldous, C. R. (2009). The Genesis of New Ideas: Models, Feeling and Solutions. In B. Matthews \& T. Gibbons (Eds.), The Process of Research in Education Shannon (pp 338-370). Adelaide: Research Press.

Aldous, C. R. (2012). Creativity, Problem Solving and Feeling, In Nobert M. Seel (Ed.), Encyclopedia of the Sciences of Learning, (Part 3, pp 843-846). New York, NY: Springer Science.

Aldous, C. R. (2014). Attending to Feeling: It May Matter More than You Think. Creative Education 5, 780-790. https://doi.org/10.4236/ce.2014.510091

Aldous, C. R. (2015). From Where Do New Ideas Come? Unlocking the Mystery of Creativity and the Solving of Novel Mathematics Problems. Adelaide: UNESCO APNIEVE Australia Publishing.

Amabile, T. M. (1996). Creativity in Context: Update to the Social Psychology of Creativity. Boulder, CO: Westview.

Arieti, S. (1976). Creativity: The Magic Synthesis. New York: Basic Books.

Australian Mathematics Trust (AMT) (2001). Mathematics Challenge for Young Australians: Teacher's Reference Book for Primary, Junior \& intermediate Stages: An Activity of the Australian Mathematics Olympiad Committee: A Subcommittee of the Australian Mathematics Trust in Association with the Australian Academy of Science and the University of Canberra. Canberra: AMT Publishing.

Cohen, J. (1992). A Power Primer. Psychological Bulletin, 112, 155-159. https://doi.org/10.1037/0033-2909.112.1.155 
Cropley, A. J. (2001). Creativity in Education and Learning a Guide for Teachers and Educators. London: Kogan Page.

Cropley, A., \& Cropley, D. (2008). Resolving the Paradoxes of Creativity: An Extended Phase Model. Cambridge Journal of Education, 38, 355-373. https://doi.org/10.1080/03057640802286871

Damasio, A. R. (1994). Descartes' Error: Emotion Reason and the Human Brain. London: Papermac.

Damasio, A. R. (1999). The Feeling of What Happens. London: William Heinemann.

Das, J. P. (2003). A Look at Intelligence as Cognitive Neuropsychological Processes: Is Luria still Relevant? Japanese Journal of Special Education, 40, 631-647. https://doi.org/10.6033/tokkyou.40.631

Dehaene, S., Spelke, E., Pinel, P., Stanescu, R., \& Tsivkin, S. (1999). Sources of Mathematical Thinking: Behavioural and Brain-Imaging Evidence. Science Magazine, 284, 970-977.

Dold, A., \& Eckmann, B. (1977). Structural Stability: The Theory of Catastrophes and Applications in the Sciences. New York, NY: Springer-Verlag.

Epstein, S. (1994). Integration of the Cognitive and the Psychodynamic Unconscious. American Psychologist, 49, 709-724.

https://doi.org/10.1037/0003-066X.49.8.709

Epstein, S. (1998). Constructive Thinking the Key to Emotional Intelligence. Westport, CT: Praeger.

Finke, R. A., Ward, T. B., \& Smith, S. M. (1992). Creative Cognition: Theory, Research and Application. Cambridge, MA: MIT Press.

Gallate, J., Wong, C., Ellwood, S., Roring, R. W., \& Snyder, A. (2012). Creative People Use Non-Conscious Processes to Their Advantage. Creativity Research Journal, 24, 146-151. https://doi.org/10.1080/10400419.2012.677282

Ghiselin, B. (1963). The Creative Process and Its Relation to the Identification of Creative Talent. In C. W. Taylor, \& F. Barron (Eds.), Scientific Creativity: Its Recognition and Development. New York, NY: John Wiley \& Sons.

Gleick, J. (1987). Chaos. New York, NY: Viking.

Guilford, J. P. (1967). The Nature of Human Intelligence. New York, NY: McGraw Hill.

Hadamard, J. (1945). An Essay on the Psychology of Invention in the Mathematical Field. Princeton, NJ: Princeton University Press.

Hu, L. T., \& Bentler, P. M. (1999). Cutoff Criteria for Fit Indexes in Covariance Structure Analysis: Conventional Criteria versus New Alternatives. Structural Equation Modeling: A Multidisciplinary Journal, 6, 1-55. https://doi.org/10.1080/10705519909540118

Immordino-Yang, M. H., \& Faeth, M. (2010). The Role of Emotion and Skilled Intuition in Learning. In D. A. Sousa (Ed), Mind Brain and Education (pp. 69-84). Bloomington: Solution Tree Press.

Isaksen, S. G., \& Parnes, S. J. (1985). Curriculum Planning for Creative Thinking and Problem Solving. Journal of Creative Behavior, 19, 1-29. https://doi.org/10.1002/j.2162-6057.1985.tb00400.x

Jeffries, D. A. (2011). Cognitive and Non-Cognitive Processes: Students' Perceptions about and Employment of Approaches to Novel Mathematics Problem Solving. Honors, Thesis, Adelaide: The Flinders University of South Australia.

Jeffries, D., \& Aldous, C. R. (2016). Recognising Intimation: An Affective Reality in the Act of Creation. In J. Orrell, \& D. D. Curtis (Eds.), Publishing Higher Degree Research 
Making the Transition from Student to Researcher (pp. 105-114). Rotterdam: The Netherlands Sense Publishers.

https://doi.org/10.1007/978-94-6300-672-9_11

Jöreskög, K. G. (1969). A General Approach to Confirmatory Maximum Likelihood Factor Analysis. Psychometrika, 34, 183-202. https://doi.org/10.1007/BF02289343

Kahneman, D. (2011). Thinking Fast and Slow. Maryborough: Allen Lane.

Kline, R. B. (1998). Principles and Practice of Structural Equation Modeling. New York, NY: The Guilford Press.

Lubart, T. (2001). Models of the Creative Process: Past Present and Future. Creativity Research Journal, 13, 295-308. https://doi.org/10.1207/S15326934CRJ1334_07

Miller, A. I. (1992). Scientific Creativity: A Comparative Study of Henri Poincare and Albert Einstein. Creativity Research Journal, 5, 385-418.

https://doi.org/10.1080/10400419209534454

Mumford, M. D., Mobley, M. I., \& Uhlman, C. E. (1991). Process Analytic Models of Creative Capacities. Creativity Research Journal, 4, 91-122. https://doi.org/10.1080/10400419109534380

Muthén, L. K., \& Muthén, B. O. (2010). Mplus: Statistical Analysis with Latent Variables User's Guide (Version 6). Los Angeles, CA: Authors.

Muthén, L. K., \& Muthén, B. O. (2012). Mplus: Statistical Analysis with Latent Variables User's Guide (Version 7). Los Angeles, CA: Authors.

Muthén, L. K., \& Muthén, B. O. (2017). Mplus: Statistical Analysis with Latent Variables: User's Guide (Version 8). Los Angeles, CA: Authors.

Osborn, A. F. (1953). Applied Imagination: Principles and Procedures of Creative Thinking. New York, NY: Scribner.

Patrick, C. (1938). Scientific Thought. Journal of Psychology, 5, 55-83. https://doi.org/10.1080/00223980.1938.9917552

Razak, N. A., Keeves, J. P., \& Darmawan, I. G. N. (2014). Developing a Modelling Approach and the Examination of Models. In I. G. N. Darmawan, \& C. R. Aldous (Eds.), The Processes of Change on Learning Literacy and Numeracy in South Australian Primary Schools. Adelaide: Shannon Research Press.

Rossman, J. (1931). The Psychology of the Inventor. Washington: Invention Publications.

Runco, M. A., \& Chand, I. (1995). Cognition and Creativity. Educational Psychology Review, 7, 243-267. https://doi.org/10.1007/BF02213373

Sellin, N., \& Keeves, J. P. (1997). Path Analysis with Latent Variables. In J. P. Keeves (Ed.), Educational Research, Methodology, and Measurement: An International Handbook (pp. 633-640). Oxford: Pergamon Press.

Shaw, M. P. (1989). The Eureka Process: A Structure for the Creative Experience in Science and Engineering. Creativity Research Journal, 2, 286-298.

https://doi.org/10.1080/10400418909534325

Singh, S. (2011). Fermat's Last Theorem: The Story of a Riddle that Confounded the World's Greatest Minds for 358 Years. London: The Folio Society.

Sloman, S. A. (1996). The Empirical Case for Two Systems of Reasoning. Psychological Bulletin, 119, 3-22. https://doi.org/10.1037/0033-2909.119.1.3

Sternberg, R. J. (2006). The Nature of Creativity. Creativity Research Journa1, 18, 87-98. https://doi.org/10.1207/s15326934crj1801_10

Vinacke, W. F. (1952) The Psychology of Thinking. New York: McGraw-Hill.

Wallas, G. (1926). The Art of Thought. London: Jonathan Cape Ltd. 
Submit or recommend next manuscript to SCIRP and we will provide best service for you:

Accepting pre-submission inquiries through Email, Facebook, LinkedIn, Twitter, etc. A wide selection of journals (inclusive of 9 subjects, more than 200 journals)

Providing 24-hour high-quality service

User-friendly online submission system

Fair and swift peer-review system

Efficient typesetting and proofreading procedure

Display of the result of downloads and visits, as well as the number of cited articles Maximum dissemination of your research work

Submit your manuscript at: http://papersubmission.scirp.org/

Or contact ce@scirp.org 